\title{
Development of an Ontology-Directed Signal Processing Toolbox
}

\author{
Award No. DE-SC0004686
}

Principal Investigator: Dr. Stephen W. Lang

The Aptec Group

27 May 2011 


\section{Executive Summary}

This project was focused on the development of tools for the automatic configuration of signal processing systems. The goal is to develop tools that will be useful in a variety of Government and commercial areas and useable by people who are not signal processing experts.

In order to get the most benefit from signal processing techniques, deep technical expertise is often required in order to select appropriate algorithms, combine them into a processing chain, and tune algorithm parameters for best performance on a specific problem. Therefore a significant benefit would result from the assembly of a toolbox of processing algorithms that has been selected for their effectiveness in a group of related problem areas, along with the means to allow people who are not signal processing experts to reliably select, combine, and tune these algorithms to solve specific problems.

Defining a vocabulary for problem domain experts that is sufficiently expressive to drive the configuration of signal processing functions will allow the expertise of signal processing experts to be captured in rules for automated configuration. In order to test the feasibility of this approach, we addressed a lightning classification problem, which was proposed by DOE as a surrogate for problems encountered in nuclear nonproliferation data processing.

We coded a toolbox of low-level signal processing algorithms for extracting features of RF waveforms, and demonstrated a prototype tool for screening data. We showed examples of using the tool for expediting the generation of ground-truth metadata, for training a signal recognizer, and for searching for signals with particular characteristics.

The public benefits of this approach, if successful, will accrue to Government and commercial activities that face the same general problem - the development of sensor systems for complex environments. It will enable problem domain experts (e.g. analysts) to construct signal and image processing chains without the aid of signal processing experts. Thus enabled, problem domain experts will be able to work more quickly and produce better quality work. 


\section{Goals and Accomplishments}

Several recent research efforts have investigated the use of ontological languages in building tools to assist users who lack image processing expertise to efficiently accomplish image and video processing tasks. A component of proposed solutions has been the definition of a signal description ontology that is comprehensible to users, effective in describing signals so that they may be usefully distinguished from one another, and composed of classes and properties whose existence and values can be reliably extracted from raw data by automatic algorithms.

A key research question to be investigated under this SBIR phase 1 program was whether an RF signal concept ontology could be devised that is both accessible to domain experts and which also provides a useful bridge to underlying signal processing algorithms. As in the image processing efforts, such an ontology would provide a "language" in which an analyst could describe a signal of interest. Having the terms of the language tied to underlying signal processing algorithms would then allow a recognition algorithm to be constructed in an automated fashion.

A lightning classification problem was proposed by DOE as a surrogate for problems encountered in nuclear nonproliferation data processing. Electric field change waveforms captured by the Los Alamos LASA sensor network were provided to us, together with classifications produced by an algorithm developed at Los Alamos. We developed a library of signal processing functions to process these waveforms and extract potential classification features. Simple signal recognizers that could be conveniently expressed using OWL 2 ontology language constructs were developed, as well as more complex recognizers capable of better performance.

The use of these components was demonstrated in two applications:

- A conventional classifier development scenario, in which a principal cost is the creation of ground truth metadata that is needed for classifier training and testing. This is often a laborintensive operation and few off-the-shelf tools are available to aid productivity. We demonstrated a prototype "ground-truth editor" which automatically decomposes signals into primitives described in an ontology, runs simple recognizer algorithms to suggest values for the ground-truth metadata, and presents information to allow the user to verify the suggested metadata in a time-efficient manner.

- A simple search scenario in which a user is searching for examples of a signal that is similar to a given signal. We demonstrated a search tool that extracts features from the given signal and allows the user to search for other signals with similar feature values.

For the surrogate problem it appears that generically defined features can result in a reasonable level of performance on the lightning data and that a description of a waveform in terms of these features, as well as ground truth metadata, and metadata describing the structure of the LASA data is easily capable of representation in the OWL 2 ontology language.

This conclusion provides motivation for a system architecture in which signal metadata is stored in the form of ontologies, and various tools operate on this data to perform functions such as ground truthing, classifier training, signal categorization and classification, and search. OWL 2 is attractive for a number of reasons: it has standardized syntax and file formats, it supports translation between different ways of describing the similar data as well as descriptions that change over the course of system development, and because it offers the promise of implementing some functions using general-purpose reasoners or rule engines instead of as special purpose code. 


\section{Screening Tool}

Several recent research efforts have investigated the use of ontological languages in building tools to assist users who lack image processing expertise to efficiently accomplish image and video processing tasks. ${ }^{1}$ A key research question to be investigated under this SBIR phase 1 program was whether an RF signal concept ontology could be devised that is both accessible to domain experts and which also provides a useful bridge to underlying signal processing algorithms. It was proposed to demonstrate this by building a prototype signal screening tool which would be capable of searching through a database for signals that fit a given description.

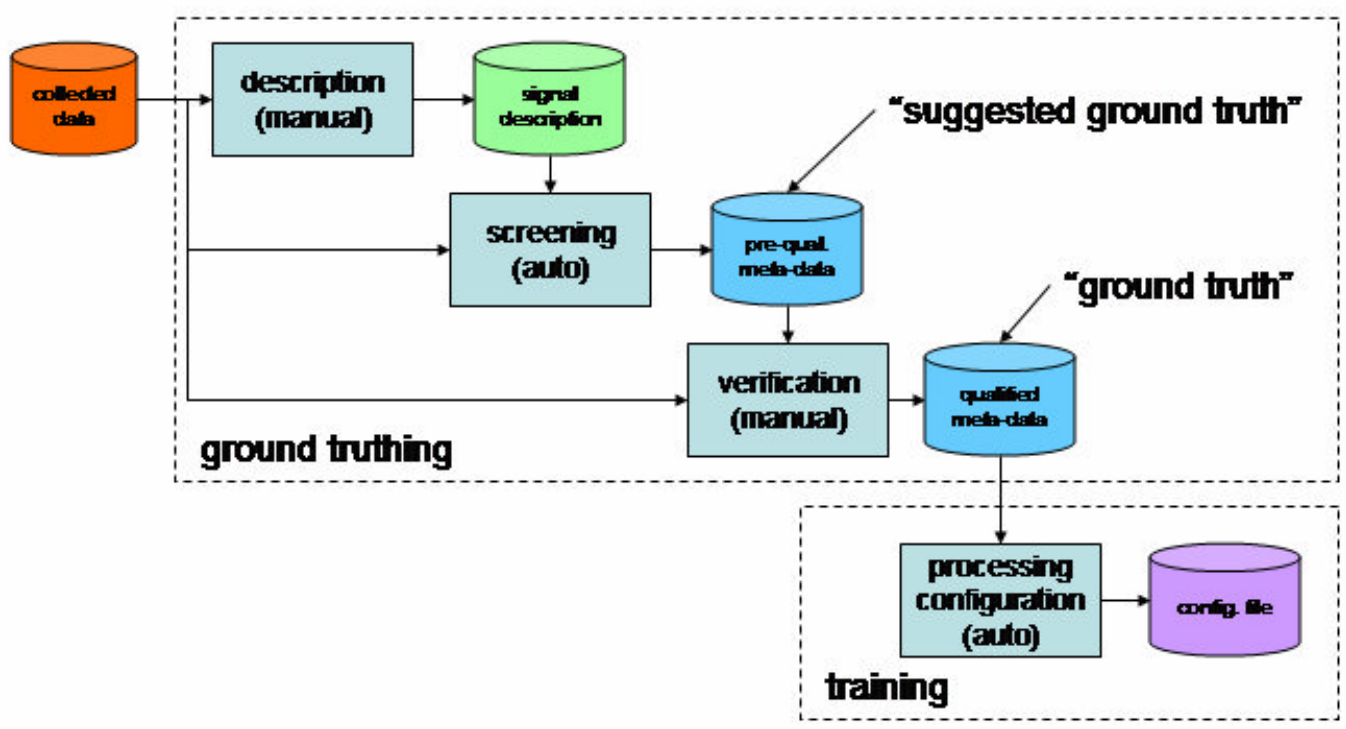

Figure 1. Screening tool supports a workflow that leads from a manual description of a signal-of-interest to the automatic configuration of a recognizer for that signal.

The workflow supported by such a tool and illustrated in Figure 1 comprises the following steps:

1. Description: the user describes a signal type or types in terms of classes and properties contained in an ontology, specifically in this case an "RF Concepts" ontology. The result of this step is a signal description.

2. Screening: the tool is able to automatically search or screen a database of collected signals for examples that fit the descriptions created by the user. The result of this step is "prequalified" metadata associated with each signal instance. It is "pre-qualified" in the sense that it has not yet been subjected to manual verification.

3. Verification: The tool presents the user with the pre-qualified meta data for each signal, together with an appropriate plot or other visualization of the signal. The user may confirm or modify the automatically-generated metadata. The result of this step is "qualified" metadata, a.k.a. "ground truth data".

4. Processing configuration: The tool uses the qualified metadata to automatically configure a processing chain to recognize one or more signal types.

\footnotetext{
${ }^{1}$ A summary of these research efforts is given by Nadarajan (2010).
} 
This workflow supports the conventional classifier development task, in which data is collected, groundtruth metadata is attached, features are selected and extracted, and classifier algorithms are trained and tested. The component functions also support other tasks, for example searching for signals that are similar to a given signal.

\section{Surrogate Problem}

An initial meeting was held with DOE on 29 September 2010 to kick off the program. A principal result of the meeting was the definition of a surrogate signal recognition problem, success in which would build confidence in the approach. Two options were discussed at the meeting. Both involve lightning data. The advantage in using lightning data is that it is unclassified, available through LANL (Los Alamos National Laboratory), and there is published research that describes how different types of lightning can be discriminated from one another in the data. Two options were discussed:

- Option 1: A model of a signal of interest (SOI) may be available from LANL. The problem posed would be to discriminate this SOI from lightning.

- Option 2: This option does not require definition of a separate signal of interest. The problem posed would be to discriminate different types of lightning from one another.

Option 2 was eventually chosen and we were put in touch with Tim Hamlin, a lightning expert at LANL, who has been very helpful in providing data, references, and explanations.

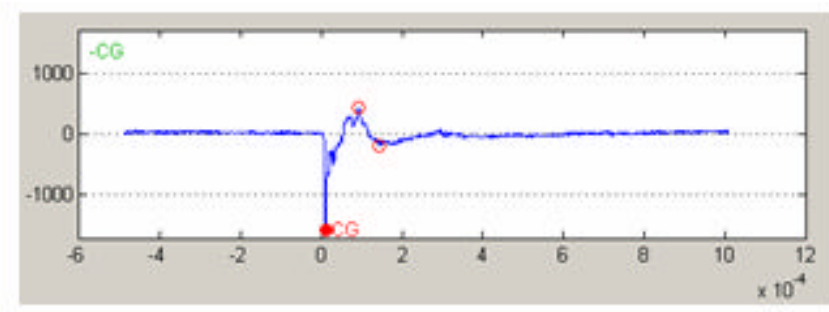

$-\mathbf{C G}$
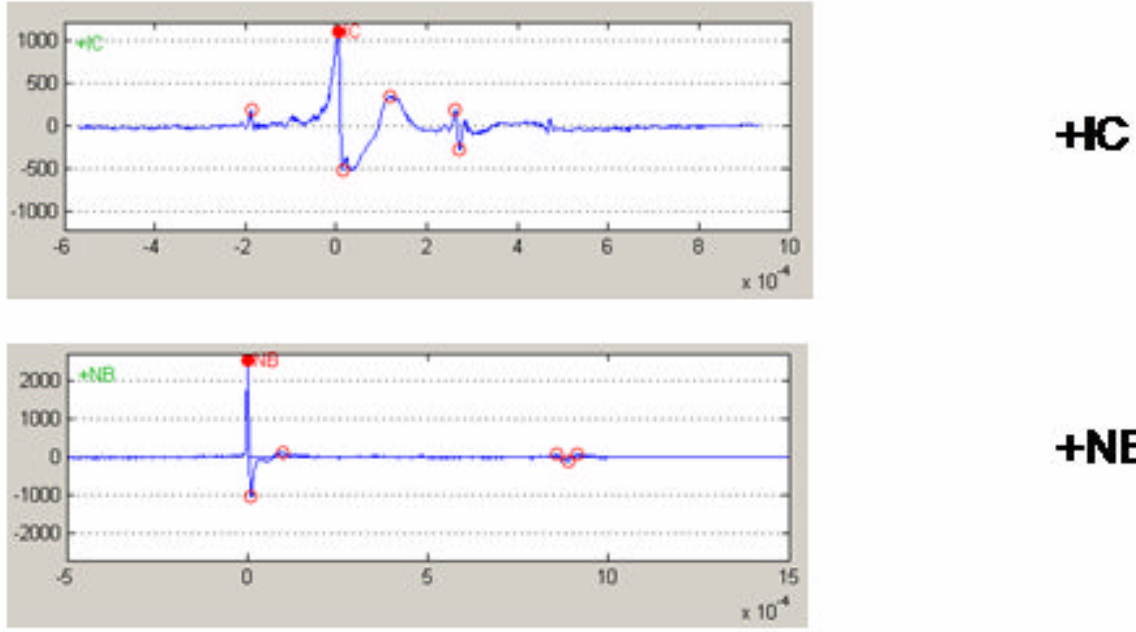

\section{$+\mathbf{N B}$}

Figure 2. Representative field change waveforms associated with the three types of lightning we focused on: cloud-to-ground (CG), intracloud (IC), and narrow-bipolar (NB). 
A description of the lighting classification problem is available in the published literature. See for example Hamlin $(2007,2009)$ and Shao (2006). In this effort we focused on the two major lightning type: cloud-to-ground (CG), intracloud (IC), together with a type of intracloud known as narrow-bipolar (NB).

Dr. Hamlin provided us with data collected from The Los Alamos Sferic Array (LASA)., specifically from a half-dozen stations located generally in the Great Plains region of the US. At each station, a charge amplifier connected to a metal plate is sensitive to the local electric field. The output of the amplifier is high-passed filtered with about a $1 \mathrm{~ms}$ time constant and digitized at $1 \mathrm{MHz}$ to produce an "electric field change" waveform. $1.5 \mathrm{~ms}$ of waveform was recorded at each station triggered when by an event.

Representative waveforms recorded by the stations are shown in Figure 2. CG discharges are characterized by a leading excursion with a fast rise and a slow decay, while IC discharges exhibit a slow rise and a fast decay. NB discharges are characterized by a fast rise and a fast fall. A polarity (+/-) is assigned to each event according to the sign of the leading excursion.

The data we received from LANL had been processed in order to geolocate the discharge event using time-difference-of-arrival. As a byproduct of this processing, a "feature time" was available for each station, which recorded the estimated time location of the discharge within each waveform. The relative feature times between two stations represented the estimated time-difference-of arrival associated with the geolocation. In addition, we had available the type classification automatically produced by an algorithm developed at LANL.

The feature time was not always accurate enough to unambiguously identify the correct peak in a waveform by itself. However the relative feature times between two stations were accurate and the location of a peak relative to the feature time proved to be an important feature in selecting the correct peak to classify.

\section{Description Features and Schema}

The first part of our effort involved formulating a set of signal processing functions whose purpose was to extract various recognition features from a signal. The signal processing chain that we assembled for this purpose is illustrated in Figure 3.

In processing data from a given discharge event, only data from stations within $750 \mathrm{~km}$ of the event was used. In doing so we followed the observed behavior of the LANL algorithm, which produced no type classification when all stations were more than $750 \mathrm{~km}$ from the event.

The first step in the process was to detrend the waveform from each station waveform associated with a geolocated discharge event. A very large trend is often present in the data because the high pass filter time constant is relatively large compared to the record length. Detrending was accomplished by fitting an subtracting a first order polynomial from each waveform.

The next step was to stack the individual waveforms from each station to produce a single composite waveform. This was accomplished by time-shifting each waveform to place the feature-time at zero, and them computing a weighted sum. The weight applied to each waveform was inversely proportional to the estimated noise variance in the waveform. 


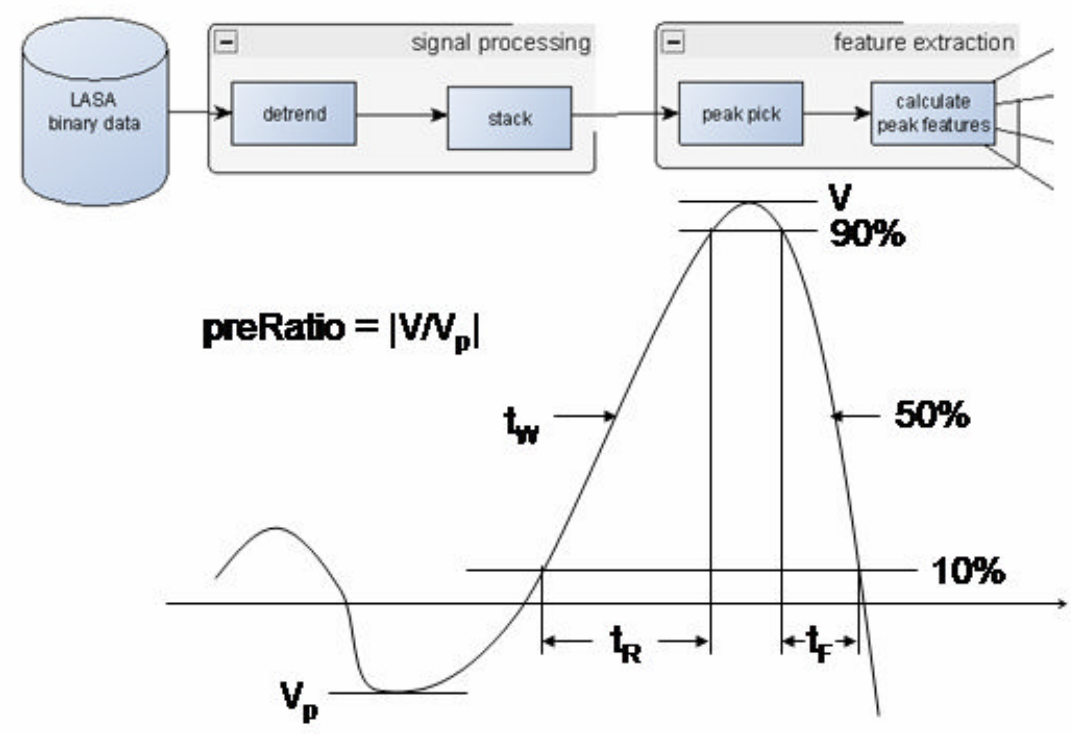

Figure 3. Signal processing chain used to compute recognition features.

After stacking, a peak picking algorithm was used to locate all peaks in the composite waveforms that exceeded a threshold SNR. Finally, various features of these peaks were calculated. The specific features calculated for each peak were:

1. fall time - the time it takes the signal to fall from $90 \%$ of its peak to $10 \%$.

2. peak time - the time at which the peak value is attained. This is measured relative to the feature time provided by the geolocation algorithm.

3. peak value - the value of the waveform at the peak time

4. preRatio - the absolute ratio of the peak value to the peak value of the preceding peak. A high preRatio is indication that a peak is the leading excursion associated with a discharge event.

5. rise time - the time it takes the signal to rise from $10 \%$ of its peak to $90 \%$.

6. SNR - the ratio of the peak value to the estimated noise standard deviation, where the entire waveform is used to estimate the noise level.

7. SNRPre - the ratio of the peak value to the estimated noise standard deviation, where the part of the waveform preceding the peak time is used to estimate the noise level.

8. width - the width of the peak, measured at $50 \%$ of the peak value.

These features, together with the other information provided in a LASA event recoded, can be described using a pair of ontologies as illustrated in Figure 4. The first step in the workflow illustrated by Figure 1 is for the user to describe a signal or signals in terms of pulse properties in the RF Concepts ontology. 


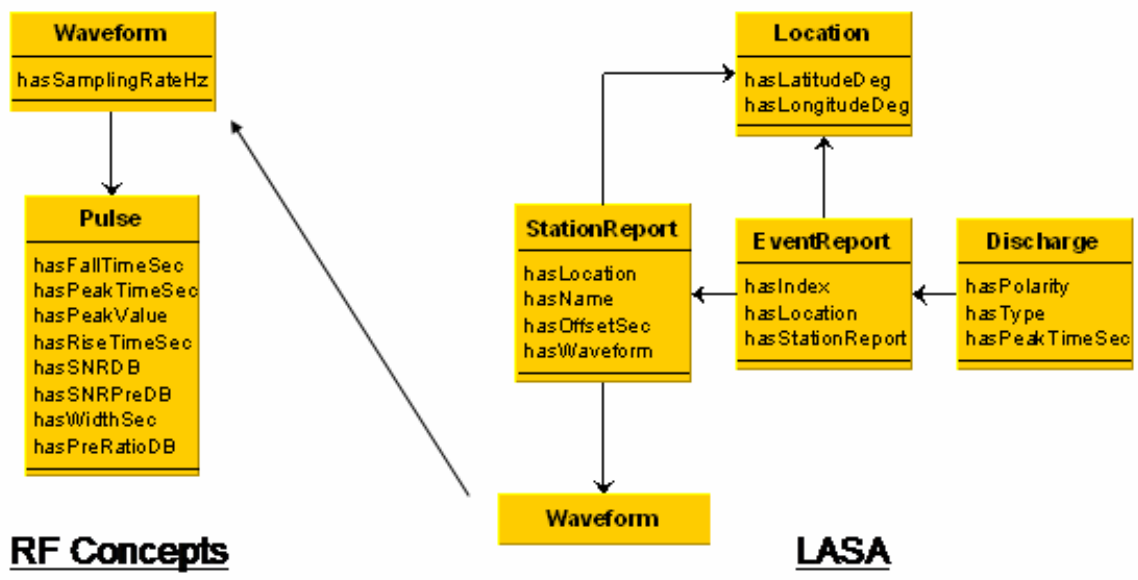

Figure 4. An RF Concepts ontology (Left) captures application-independent features of a time-domain waveform composed of "pulses". A LASA ontology (Right) captures the application-dependent structure of data from the LASA array and the desired "Discharge" product of a signal recognizer.

\section{Simple Recognizers}

The second step in the process illustrated by Figure 1 is to use simple recognizers to generate prequalified metadata, i.e. the properties listed under "Discharge" in Figure 4. This was accomplished by implementing a collection of simple recognizers that examine each peak in the composite waveform for properties that matched the user-input description of a single signal type, as shown in Figure 5. If more than one recognizer fires on a given waveform, of if one recognizer fires on more than one peak, a simple voting procedure that gives preference to the largest peak is used to select a single output.

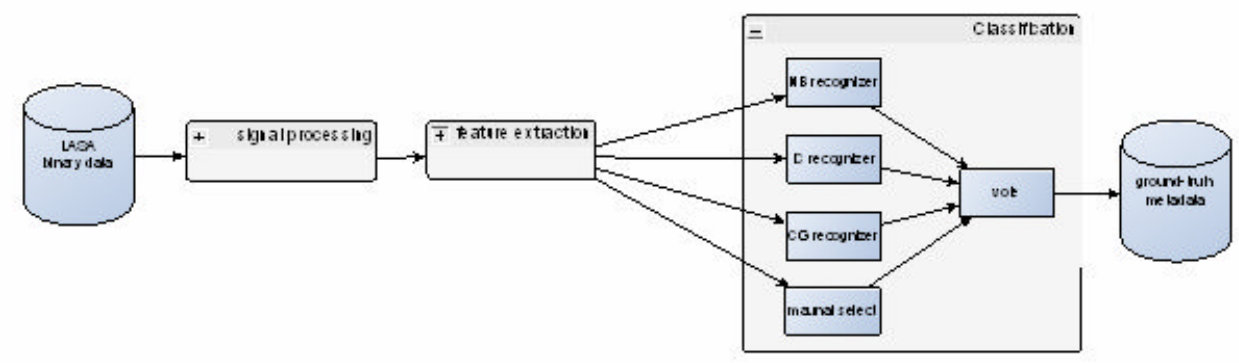

Figure 5. A collection of simple recognizers support automatic screening.

The decision region of each simple recognizer is a hyperrectangle, as shown in Figure 6 . This is the result of testing each peak feature against an upper and/or lower numerical limit. Such a recognizer is capable of being expressed using datatype restrictions in the OWL 2 ontology language (Motik et al. 2009) or using SWRL built-ins (Horrocks et al. 2004) and therefore executable by an OWL reasoner or a SWRL rule engine. 


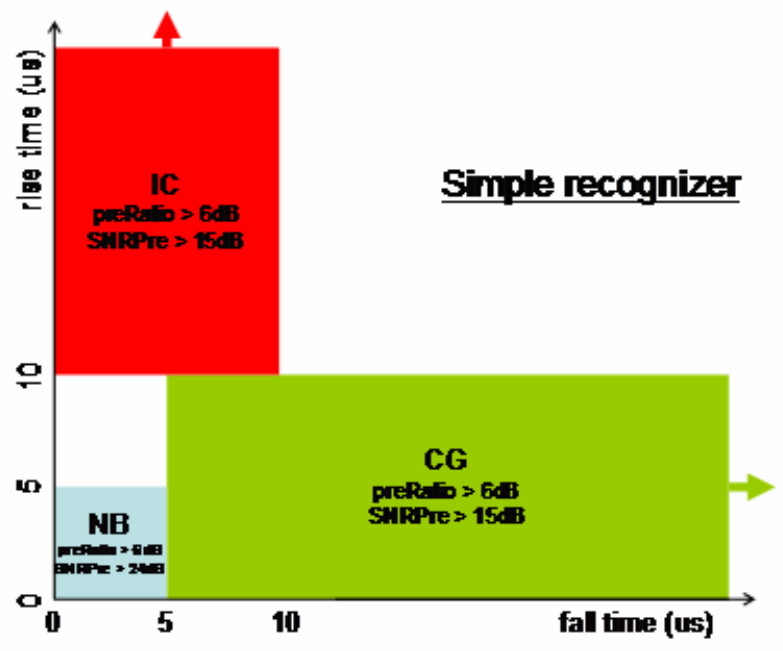

Figure 6. Each simple recognizer has a decision region described by a hyperrectangle in feature space.

More complex decision regions are theoretically expressible in these languages using union and intersection relationships; however we expect that it would be difficult for users to manually compose complex decision regions in this way. Fortunately, simple recognizers with hyperrectangle decision regions appear to have good enough performance to be useful in generating pre-qualified metadata for the lightning classification problem.

\section{Ground Truthing}

The final step in the ground-truthing segment of the Figure 1 workflow is manual verification of the prequalified metadata to produce qualified-meta data. Another way to look at the workflow is to view the preceding steps as aids aiming at improving the throughput of a manual ground-truthing process.

Modern signal and image analysis and classification systems must not only process raw sensor data into high quality multi-channel time series waveforms, images, or videos, but they must also identify and classify objects within these waveforms or images. Achieving good performance often requires the use of large quantities of "ground-truth" data, i.e. sensor data for which the correct answers are known and are available in a machine-processable form. Model parameter estimation, classifier training, performance evaluation, and root-cause failure analysis are all tasks that use ground-truth data. However creating, maintaining, and providing efficient human and machine access to annotated data sets can be a very time consuming task.

By presenting suggested metadata to the user, generated by simple recognizers, the hope is that the user can dispatch a large percentage of events by clicking on a single button that accepts the suggested metadata and increments to the next event (Figure 7). In order to enable this there must also be a GUI that presents a visualization of the data that gives the user what information is necessary to verify the suggestion. In the lightning application this is provided by providing a display of the composite "Stack" waveform (backed up by displays of individual station waveforms, together with an identification of significant peaks and the features measured for each peak. The suggested peak is indicated by a filled circle that is annotated with lightning type. 
The simple recognizers are represented in the GUI by tab panels on the right. Each tab panel allows the user to set parameters controlling the boundaries of the recognizer's decision region.

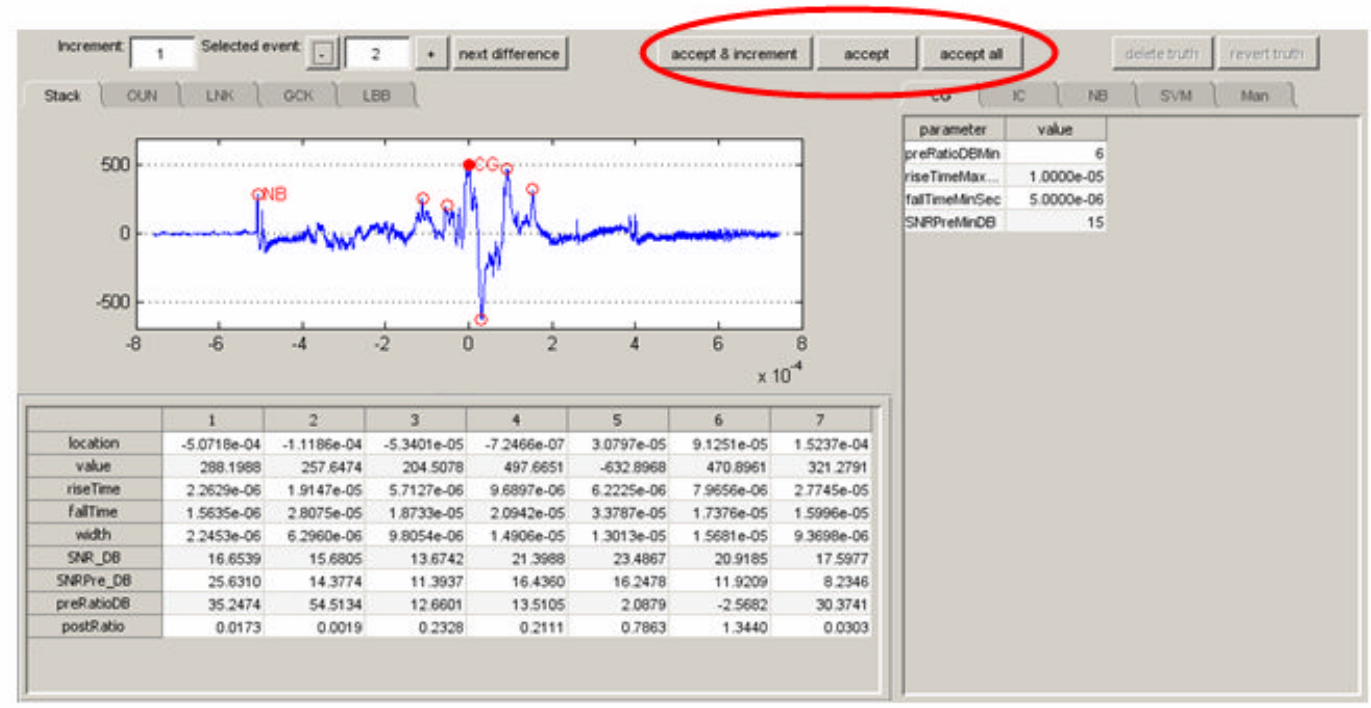

Figure 7. The user can accept metadata for an event that is suggested by the simple recognizers by clicking on a single button.

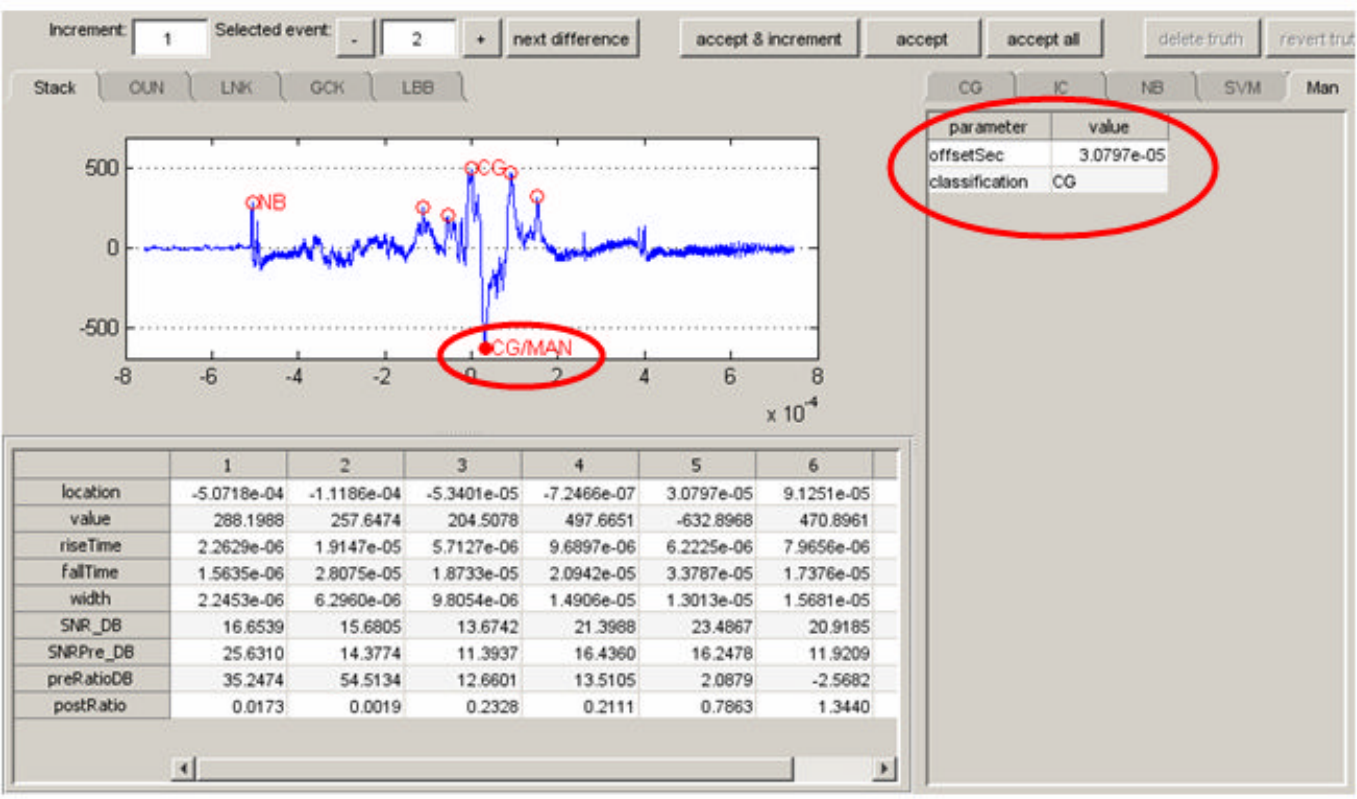

Figure 8. Suggested metadata can be overridden by right-clicking on any circled peak and selecting a lightning type from a pop-up menu. The selection is remembered and associated with the event index.

If the suggested metadata is judged to be incorrect the GUI should provide an efficient means of correction. In the lightning application this is provided by a context menu that pops-up when the user right-clicks on any circled peak. The user selects a lightning type from the list, which together with the peak that was clicked on fully determines all the metadata for this event. The manual selection is remembered by a "Man[ual]" pseudo signal recognizer, which associates the manually-selected metadata with the index number of the event. This recognizer is represented by a tab panel along with the other recognizers (Figure 8). Its tab panel displays the peak time and lightning type of events for 
which a manual selection has been made. Additionally, a manual selection is given priority over the output of the simple recognizers in the voting process that determines the suggest metadata, forcing the suggestion to follow manual selection where they have been made.

The ground truth data may be saved to a file in OWL 2 format and recalled for editing later.

\section{Training and SVM-Based Recognizer}

One the qualified metadata or ground truth data has been generated, it may be used to configure a recognizer that is more powerful than the simple recognizers discussed above. This recognizer shares the same signal processing chain as the simple recognizers, through the peak feature computation step. However it processes the peak information differently.

The way in which this recognizer is structured generally follows the way in which the LANL algorithm works: first it selects a particular peak, and then it performs type-classification on that peak. ${ }^{2}$ However the specifics of the algorithm are different. In our recognizer peak detection is performed on the stacked waveform using a ranking SVM. In selecting a peak the peak time and polarity are determined. The features of the selected peak are input into a multi-class SVM, which selects a type classification (Figure 9).

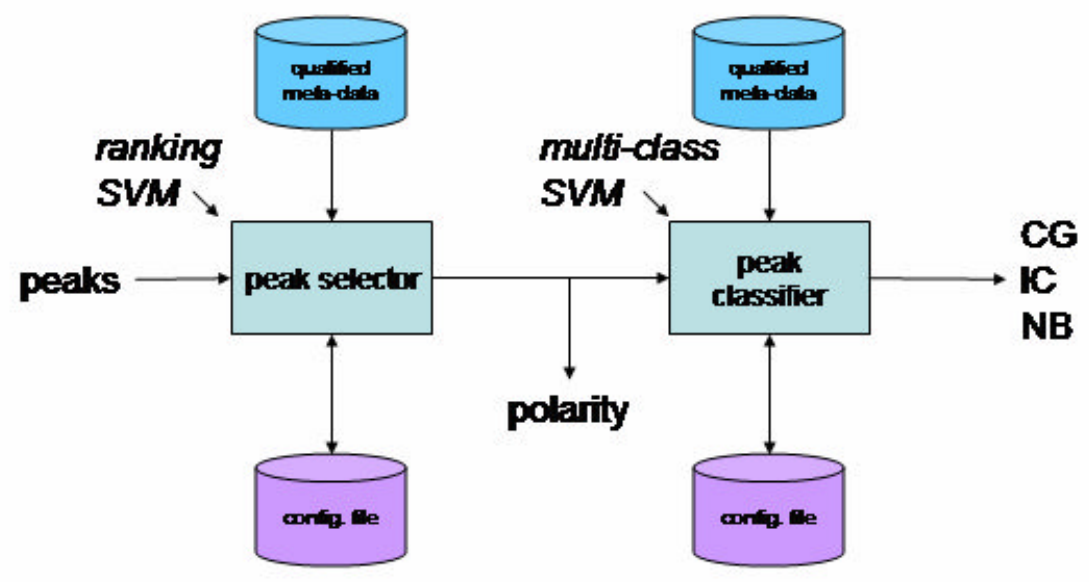

Figure 9. Final processing configuration consists of training a peak selector and a peak classifier. Both are based on support vector machines.

The two SVMs may be trained independently: the peak time property of the ground truth data is used to train the peak selector on input data consisting of the peaks in each waveform and their features. The lightning type property of the ground truth data is used to train the peak classifier SVM on input data consisting of the correct peak as indicated by the ground truth data, and the features of this peak.

Once trained, the SVM-based recognizer can be used to classify lightning waveforms with better performance than the simple classifiers.

On the GUI, the SVM recognizer is represented by its own tab panel, which allows the user to select the features to be used for peak selection and classifications. Unary transforms of the peak ranking features may be specified. Additionally, various options for the peak classifier, which was implemented

\footnotetext{
${ }^{2}$ The specific algorithm used by LANL is not fully known to us. The general structure was described to us by Dr. Hamlin.
} 
using LIBSVM, can be specified [Chang 2010]. The peaking ranking algorithm was based on Joachim's rankSVM [2002].

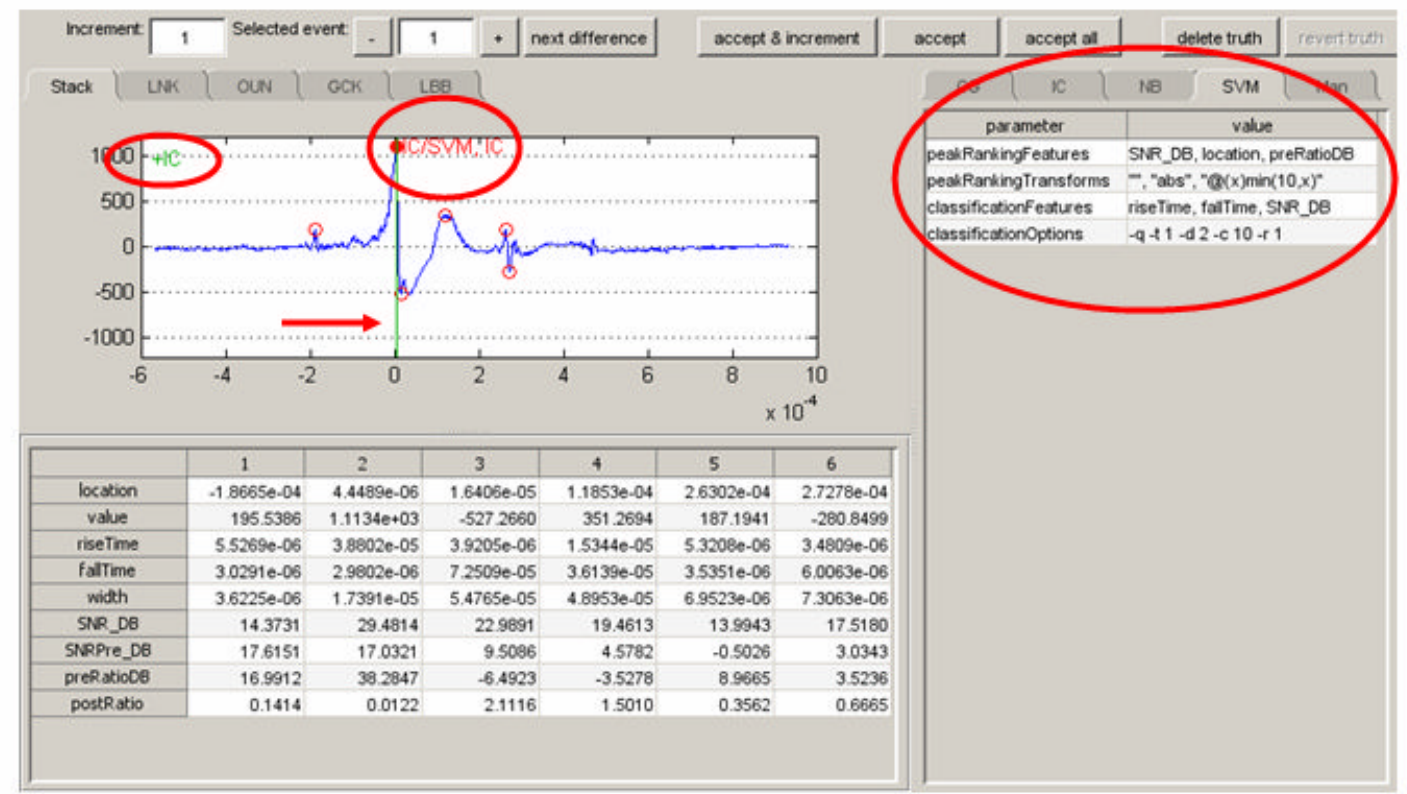

Figure 10. A tab panel on the right represents the SVM-based classifier. It allows the user to specify features to be used for peak selection and classification, as well as other options.

Once ground truth has been generated and the SVM recognizer training, the GUI appears as in Figure 10:

- The ground truth is overlaid on the plot window in green: the ground truth polarity/type is noted in the upper left, and the ground truth time is shown by the green vertical line.

- The peak selected by the peak selector is shown as a filled circle. It takes priority over the output of the other recognizers. The type classification output of the SVM recognizer noted to the right of the filled circle.

A feeling for the improved performance potential of SVMs as compared to the simple recognizers can be had by comparing the peak classification SVM decision regions in Figure 11 to those of the simple recognizers in Figure 6 . Whereas the simple recognizers are constrained to have decision regions describer by hyperrectangles aligned with the coordinates axes, SVMs can achieve more general decision regions. In this case the SVM decision regions lie in a 3-dimensional feature space of rise time, fall time, and SNR. Figure 11 shows 32 -dimensional slices through this feature space, at SNR values of 30,35 , and $40 \mathrm{~dB}$. 

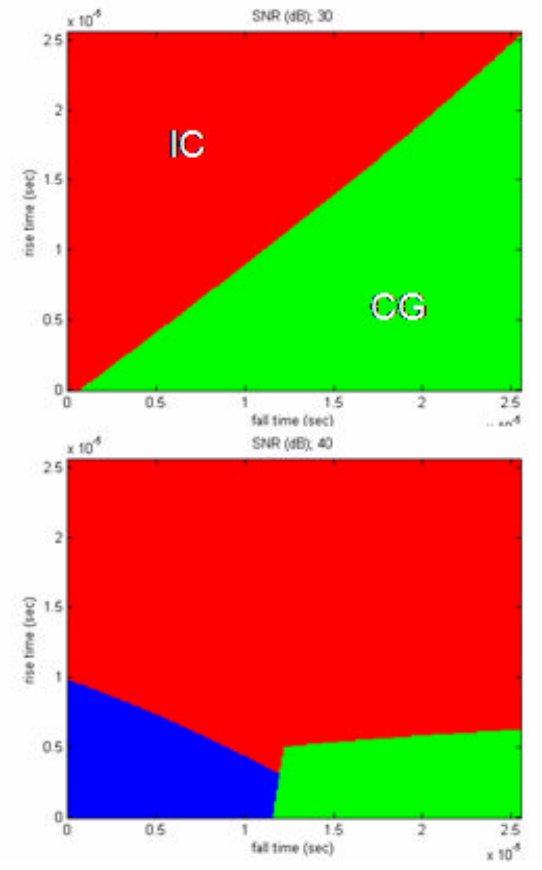

Figure 11. Decision regions of the SVM-based recognizer.

These decision regions were produced by manually ground truthing every tenth event in the provided data set, and then training the SVM classifier. In the interests of time, nominal values were chosen for the peak classifier SVM penalty factor and radial basis function width. ${ }^{3}$

\section{Lighting Type-Classification Example}

An exercise was conducted to see how well our processing chain could replicate the output of the LANL algorithm in classifying discharges as being CG, IC, or NB events. (Events for which the LANL algorithm produced type 'FP' or '??" were discarded for this exercise.)

The data set was divided into two sets: a training set consisting of events with odd indices, and a test set consisting of events with even indices. The LANL algorithm type and polarity decisions were used as truth data.

The training set was further processed in order to attach a peak time to each event, which was unfortunately not recorded in the LANL data. To do this we used the prototype software described above to manually assign a peak time to each training instance, resulting in 1333 fully truthed training instances.

The LIBSVM training algorithm was modified so that weighting could be applied to even out the false alarm probability for each class. After SVM training, the output of the SVM was compared, in type and polarity, to the LANL output on the test set, consisting of even index events. There were 1397 instances in the test set.

\footnotetext{
${ }^{3}$ With these fixed, training consists of solving a convex optimization problem, which was fast enough to implement interactively for the surrogate problem. However finding the optimal penalty factor and basis function width would require a more extensive search which would better be implemented as an off-line operation.
} 
Table 1. Comparison between SVM and LANL polarity decision

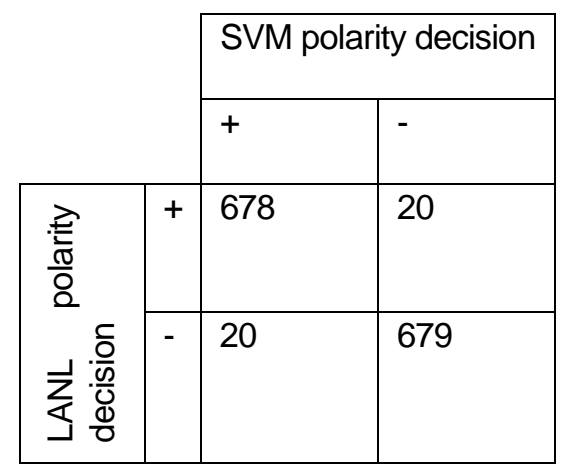

$2.9 \%$ of the polarity decisions made by the SVM recognizer are different that the LANL polarity decisions. If we assume that picking an incorrect peak leads to a $50 \%$ change of picking a different polarity, this implies that the SVM and the LANL algorithms are looking at a different peak about 5.8\% of the time.

Table 2. Comparison between SVM and LANL type decision

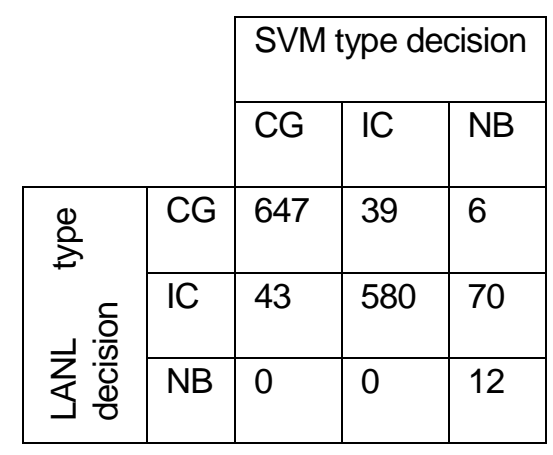

If we accept the LANL algorithm as being always correct, the type decision comparison of Table 2 implies the following:

- the probability of correct type classification of CG events is approximately $94 \%$

- the probability of correct type classification of IC events is approximately $84 \%$

- the probability of correct type classification of NB events is approximately $100 \%$

If in addition we accept that the relative frequencies of each type of event is as represented by the LANL data set, then the overall probability of correct type classification is about $89 \%$.

Some of the type classification errors are presumably due to the fact that the LANL and SVM algorithms are looking at different peaks, as indicated by the comparison of the polarity decisions above. Finally, it is known that the LANL algorithm is not always correct and that some of the waveforms are ambiguous. Based on our observations done preparing the training set, we estimate that the LANL type classification error rate is on the order of a few percent.

The level of performance given by the SVM algorithm appears reasonable, given that: 
- the LANL type classification used as truth data is itself imperfect,

- the classification features used were generic, not tailored to the specific application, and

- the SVM peak classifier was not optimized over penalty factor and basis function width.

Any effort to improve performance should address the first and last points, at minimum. This would require having an expert review the ground truth data to correct mistakes in the LANL algorithm type classification and our selection of peak time. It would also require implementation of a more complete SVM training procedure that includes optimization over penalty factor and basis function width. We did not further pursue higher performance levels because it was tangential to the focus of the effort.

\section{Simple Search Example}

As a second exercise, we imagined that an analysis had an example of a signal and wished to find additional examples of similar signals. To support this task we replaced the simple recognizers described above with one for which maximum and minimum values could be specified for each peak feature. This recognizer is represented by the "Int[erval]" tab on the right hand side of the GUI in Figure 12.

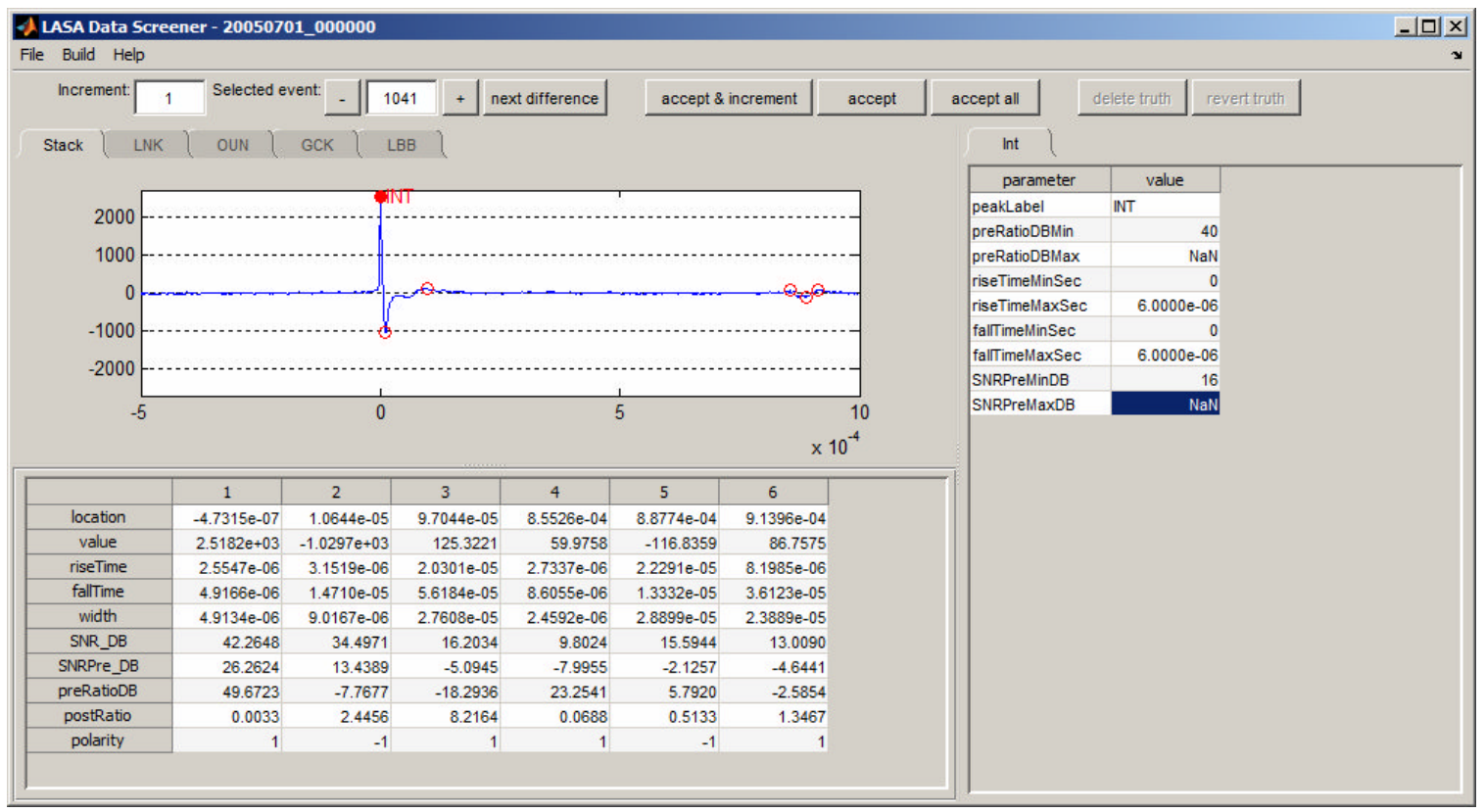

Figure 12. An interval recognizer enables the user to search for signals similar to a given signal.

In this example we open the binary data file and go to event 1041. Seeking to find similar signals., we looking at the feature values corresponding to the first peak, i.e. column 1 in the table in the lower left of the GUl, we then enter minimum and maximum values for the features on the recognizer tab. Feature value limits entered as 'NaN' will not be enforced by the recognizer.

Once the feature values are entered, clicking on the "accept all" button runs the recognizer over all events and labels all events as "INT" that have a peak whose feature values lie in the indicated interval. 
It so happens that this event, 1041, has been typed as "NB" by LANL. For the feature value limits entered, the "Int" recognizer finds 56 similar signals. These include 11 of the 23 events labeled "NB" by LANL. It also includes 45 others with short rise and fall times, which have been typed by LANL mainly as "FP" and "IC" types (other types of intracloud discharges). Only 3 of the 56 were typed as "CG" by LANL.

\section{Final Remarks}

For the surrogate problem it appears that generically defined features can result in a reasonable level of performance on the lightning data. Furthermore, a waveform description in terms of these features, as well as ground truth metadata, and metadata describing the structure of the LASA data is easily capable of representation in the OWL 2 ontology language.

This conclusion provides support for a system architecture in which signal metadata is stored in the form of ontologies, and various tools operate on this data to perform functions such as ground truthing, classifier training, signal categorization and classification, and search. OWL 2 is attractive for a number of reasons: it has standardized syntax and file formats, it supports translation between different ways of describing the similar data as well as descriptions that change over the course of system development, and because it offers the promise of implementing some functions using general-purpose reasoners or rule engines instead of as special purpose code. We envision a tool for the development of signal recognizers using large collections of collected data. Such a tool would support the Nuclear Nonproliferation mission, as well as have diverse commercial applications. Capabilities that it should support are:

- Management of large collections of data. This entails extraction of metadata data describing data sets, editing of metadata, and definition of data subsets with particular characteristics.

- Management of ground truth data. This entails extraction of ground truth data from binary files, editing of ground truth data, and definition of data subsets whose ground truth has particular properties.

- Comparing ground truth data to classifier output, scoring performance, and identifying data sets where discrepancies occur.

- Binding signal ontology properties to particular algorithm implementations used to property values.

- Generating feature vectors on identified data subsets and using them to train classifiers.

- Binding ontology properties to particular classifiers inputs and outputs

- Running trained classifier algorithms on identified data subsets.

These capabilities significantly expand on our initial concept of a screening tool. They benefit from the use of ontology languages in that the tools itself can be kept general purpose (expanding its potential field of application) and specialized to a particular application by plugging in application domain ontologies that describe data sets, features, and ground-truth/classifier-output.

Some caveats should be noted: 
- Unfortunately, the surrogate problem and the classification approach we took, which was similar to that taken by LANL, proved to be too simple to take advantage of the reasoning capability that is theoretically available. A more complex problem involving inter-relationships between different components of a signal would better test the capabilities offered by ontology languages and general purpose reasoners.

- Part of the appeal of ontology languages is the possibility of replacing application-specific code in conventional procedural languages with a combination of a general-purpose reasoning engine and some application specific data (in the form of statements or rules). However, some limited experiences with ontology reasoners and databases gained during this program suggest that care be taken relative to processing time requirements when planning the use of these components in production systems (Appendix A).

\section{Bibllography}

Chang et al., "LIBSVM: a Library for Support Vector Machines", http://www.csie.ntu.edu.tw/ cjlin/papers/libsvm.pdf, updated November 2010.

Hamlin et al., "Estimating lightning channel characteristics of positive bipolar events using intrachannel current reflection signatures", Journal of Geophysical Research, vol. 112, 2007.

Hamlin et al., "Space- and Ground-Based Studies of Lightning Signatures", chapter 13 in Lightning: Principles, Instruments and Applications, Betz (ed.), Springer, 2009.

Henss et al., "A Database Backend for OWL", Proceedings of the 5th International Workshop on OWL: Experiences and Directions OWLED 2009, Chantilly, VA, vol. 529, October 23-24, 2009.

Horrocks et al., SWRL: a Semantic Web Rule Language Combining OWL and RuleML, http://www.w3.org/Submission/SWRL/, 2004.

Horridge and Bechhofer, "The OWL API: A Java API for OWL Ontologies", Semantic WebInteroperability, Usability, Applicability, February 2011.

Hsu et al., "A Practical Guide to Support Vector Classification", http://www.csie.ntu.edu.tw/ cjlin/papers/guide/guide.pdf, updated April 15, 2010.

Joachims, "Optimizing Search Engines using Clickthrough Data", Proc. ACM Conf. On Knowledge Discovery and Data Mining (KDD), 2002.

Motik et al. (eds.), OWL 2 Web Ontology Language Structural Specification and Functional-Style Syntax, http://www.w3.org/TR/OWL 2-syntax/, 2009.

Nadarajan. "Semantics and Planning Based Workflow Composition and Execution for Video Processing". Ph.D. Thesis, CISA, School of Informatics, University of Edinburgh, August 2010.

O'Connor, M. J. and Das, A. K., 2009. SQWRL: a query language for OWL. Proceedings of the $6^{\text {th }}$ International Workshop on OWL: Experiences and Directions (OWLED 2009), Chantilly, VA, 2009.

Shearer et al., "HermiT: A Highly-Efficient OWL Reasoner", Proceedings of the $5^{\text {th }}$ International Workshop on OWL: Experiences and Directions (OWLED 2008), Karlsruhe, Germany 2008. 
Shao et al., "Total Lightning Observations with the New and Improved Los Alamos Sferic Array (LASA), Journal of Atmospheric and Oceanic Technology, vol. 23, pp. 1273-1288, October 2006.

Smith et al. 2002, "The Los Alamos Sferic Array: A research tool for lightning investigations", Journal of Geophysical Research, vol. 107(D13), July 2002. 


\section{A. OWLDB v2.0 Evaluation}

\section{A.1 OWLDB Description}

OWLDB provides relational database backend support for the OWL API library. The implementation, utilizing the Hibernate library to effectively integrate with a range of SQL databases, provides access to large ontologies while maintaining a small in-memory footprint. This functionality is relevant for server based systems which need to support a number of large ontologies.

\section{A.2 Evaluation Setup}

The OWLDB 2.0 (beta 4) source was downloaded https://owldb.svn.sourceforge.net/svnroot/owldb/ and built with the included build.bat script. The included tests when then run to verify correct build and operation using a memory resident SQL database included with the OWLDB software.

MySQL Server 5.5 (community edition) was also downloaded and installed. The OWLDB example was reconfigured to use the MySQL server using the provided Hibernate configuration file setup for MySQL.

Finally, a Java application was written (using the Eclipse IDE) that creates a LASA based ontology using the "Truth" class using a configurable number of individuals with random values for properties. The following operations where then timed for both the database and standard in-memory/file versions of the ontology:

- Creation and storing the ontology

- Loading the ontology

- Getting all axioms in the otology (twice to warm the caches)

- Query for a named individual in the ontology

- Start HermiT reasoner and pre-compute inferences for the ontology

\section{A.3 Performance Results}

The tests were run using 73,000 LASA Truth individuals (365,000 axioms) executing on a $1.6 \mathrm{GHz}$ Intel Core i7 CPU with 8 GB RAM and 7200 RPM disk system running Windows 764 -bit.

The following table shows the timing results:

\begin{tabular}{lcc}
\hline Operation & \multicolumn{1}{l}{ In-memory/file Ontology } & MySQL DB Ontology \\
\hline Create and store ontology & $5 \mathrm{~s}$ & $8,100 \mathrm{~s}(2.25 \mathrm{~h})$ \\
Load ontology & $10 \mathrm{~s}$ & $21 \mathrm{~s}$ \\
\hline
\end{tabular}




\begin{tabular}{lcc}
\hline Get all axioms & $0.1 \mathrm{~s}$ & $28 \mathrm{~s}$ \\
Start HermiT and pre-compute inferences & $9 \mathrm{~s}$ & $208 \mathrm{~s}$ \\
Query for named individual & $2.7 \mathrm{~s}$ & $2.7 \mathrm{~s}$ \\
\hline
\end{tabular}

The OWLDB Java VM memory footprint during loads was a $160 \mathrm{MB}$ with 2 to $4 \% \mathrm{CPU}$ utilization. The MySQL database server memory footprint was $205 \mathrm{MB}$ with 2 to $7 \%$ CPU utilization. Disk 10 was modest with periodic flushes to disk.

The disk size for the LASA performance MySQL database was 240 MB. Each ontology database has 67 tables.

\section{A.4 Observations}

1. OWLDB classes and methods parallel the OWL API classes and methods and the OWLDB is therefore easy to understand and use (mostly OWLDB* vs OWL* class names).

2. OWLDB may be functionally behind the latest OWL version in some areas. For example, the OWLDB loadOntology method was unable to load Turtle formatted ontologies (however the easy work around was to use directly use the OWAPIL library to load the Turtle ontology and then use OWLDB to store the ontology in the database). This could gate the use of new layered software that depends on functionality or bug fixes in new versions of the OWL API.

3. The OWLDB ontology load time is quite long. Interestingly load rates improved from 25 to 45 axioms per second after 5,000 axioms were added-perhaps a dynamic caching change. Also during load operations, neither CPU load nor disk activity where near the maximums supported by the system. This suggests that optimizations may be possible.

4. The OWLDB source code borrows heavily from OWLAPI source code, is well structured, follows standard naming conventions and coding practices, and is appropriately commented (more so than most). The code includes a range of unit, functional and performance tests.

5. OWLDB has been available for a couple of years but does not yet appear to be widely used. This may eventually limit maintenance and enhancements.

\section{A.5 Conclusion}

While the OWLDB is relatively easy to use and does enable use of a relational database to reduce the memory footprint, performance considerations, especially for loading, limits OWLDB applicability to only extremely large static databases where offline database loading can be performed. Moderate and most large sized ontologies are more easily managed by using the traditional OWL API in-memory ontologies, especially with large 64-bit servers, with file based store/loads to provide persistence. 